\title{
Evaluation of COPD Longitudinally to Identify Predictive Surrogate End-points (ECLIPSE)
}

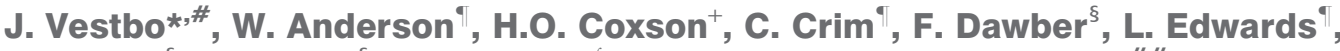 \\ G. Hagan ${ }^{\S}$, K. Knobil ${ }^{\S}$, D.A. Lomas ${ }^{f}$, W. MacNee**, E.K. Silverman ${ }^{\# \#}$ and \\ R. Tal-Singer ${ }^{\text {tT }}$ on behalf of the ECLIPSE investigators
}

ABSTRACT: Chronic obstructive pulmonary disease (COPD) is a heterogeneous disease and not well understood. The forced expiratory volume in one second is used for the diagnosis and staging of COPD, but there is wide acceptance that it is a crude measure and insensitive to change over shorter periods of time.

Evaluation of COPD Longitudinally to Identify Predictive Surrogate End-points (ECLIPSE) is a 3yr longitudinal study with four specific aims: 1) definition of clinically relevant COPD subtypes; 2) identification of parameters that predict disease progression in these subtypes; 3) examination of biomarkers that correlate with COPD subtypes and may predict disease progression; and 4) identification of novel genetic factors and/or biomarkers that both correlate with clinically relevant COPD subtypes and predict disease progression.

ECLIPSE plans to recruit 2,180 COPD subjects in Global Initiative for Chronic Obstructive Lung Disease categories II-IV and 343 smoking and 223 nonsmoking control subjects. Study procedures are to be performed at baseline, 3 months, 6 months and every 6 months thereafter. Assessments include pulmonary function measurements (spirometry, impulse oscillometry and plethysmography), chest computed tomography, biomarker measurement (in blood, sputum, urine and exhaled breath condensate), health outcomes, body impedance, resting oxygen saturation and 6-min walking distance.

Evaluation of COPD Longitudinally to Identify Predictive Surrogate End-points is the largest study attempting to better describe the subtypes of chronic obstructive pulmonary disease, as well as defining predictive markers of its progression.

KEYWORDS: Biomarkers, chronic obstructive lung disease, emphysema, genetics, lung function, natural history

hronic obstructive pulmonary disease (COPD) causes 2.75 million deaths annually, representing the fourth leading cause of death worldwide [1], and is associated with substantial morbidity [2]. COPD is a multicomponent disease, comprising emphysema in the lung parenchyma, large central airway inflammation and mucociliary dysfunction, bronchiolitis and small airway structural changes [3]. Together, these separate factors contribute to the chronic airflow limitation that characterises the condition $[3,4]$. In addition, there is evidence that systemic inflammation and extrapulmonary effects are also common in COPD, although the association between systemic inflammation and systemic manifestations of COPD is still not entirely clear.
Traditionally, both COPD diagnosis and severity evaluation have been based on spirometry $[5,6]$, and change in forced expiratory volume in one second (FEV1) over time is still the most widely accepted measure of disease progression. However, FEV1 has limitations as it measures only one aspect of the disease and is not predictive of disease progression, especially in early disease [7-9]. In addition, patients with similar FEV1 may show very different underlying pathologies, e.g. predominantly airspace disease (i.e. emphysema) or disease of the airways, as manifested by increased airway wall thickness [8]. Additionally, patients with similar FEV1 may also be of different functional status. Thus, spirometric assessment alone is insufficient for the characterisation of COPD and there is a clear
AFFILIATIONS

*Hvidoure Hospital, University of Copenhagen, Hvidovre, Denmark.

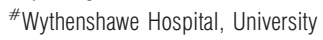
of Manchester, Manchester,

${ }^{\S}$ GlaxoSmithKline R\&D, Greenford,

fUniversity of Cambridge,

Cambridge, and

**Royal Infirmary, University of

Edinburgh, Edinburgh, UK

"GlaxoSmithKline R\&D, Research

Triangle Park, NC,

\#\#Brigham and Women's Hospital,

Boston, MA, and

"GlaxoSmithKline R\&D, King of

Prussia, PA, USA.

+University of British Columbia,

Vancouver, BC, Canada.

CORRESPONDENCE

J. Vestbo, Cardiology and Respiratory

Medicine 253, Hvidovre Hospital,

Kettegaard Alle 30, DK-2650

Hvidovre, Denmark,

Fax: 4536323716

E-mail: joergen.vestbo@

hvh.regionh.dk

Received:

August 262007

Accepted after revision:

December 212007

CLINICAL TRIALS

This study is registered at clinical Trials.gov with the identification number: NCT00292552.

STATEMENT OF INTEREST

A statement of interest for all of the authors and the study itself can be found at www.erj.ersjournals.com/ misc/statements.shtml

European Respiratory Journal Print ISSN 0903-1936 Online ISSN 1399-3003 
need for a better understanding of the conditions that comprise COPD, which is a syndrome rather than a disease.

For the assessment of response to COPD treatment, there is also a need for measures of disease progression applicable and responsive to interventional research. Although FEV1 decline has, to date, been regarded as the gold standard, different measures are likely to provide a more specific assessment of disease activity and progression within clinical subtypes of COPD. In addition, a substantial length of observation time is required for using FEV1 decline as a measure of progression, and biomarkers that could evaluate progression over a shorter period of time would be useful.

The Evaluation of COPD Longitudinally to Identify Predictive Surrogate End-points (ECLIPSE) study (registered on ClinicalTrials.gov with identifier NCT00292552 [10]; GlaxoSmithKline study code SCO104960) is a 3-yr longitudinal study with the overall objective of identifying the parameters that predict disease progression in individuals with different COPD subtypes, as well as biomarkers that may serve as surrogate end-points. The present article describes the purpose and design of the study.

\section{METHODS}

\section{Study objective}

The ECLIPSE study has the following specific aims. 1) Use of questionnaires, spirometry, exercise testing and computed tomography (CT) for the definition of clinically relevant COPD subtypes in individuals with Global Initiative for Chronic Obstructive Lung Disease (GOLD) stage II-IV COPD. 2) Identification and definition of the parameters that predict disease progression over 3 yrs in clinically relevant COPD subtypes in individuals with GOLD stage II-IV COPD. For the purposes of the present study, disease progression is defined in terms of changes in lung function variables, symptoms, exacerbation frequency, exercise capacity and airway/lung parenchymal changes on chest CT scans. 3) Measurement of known biomarkers in blood, urine, sputum and breath condensate in order to identify those that correlate with clinically relevant COPD subtypes in individuals with GOLD stage II-IV COPD and which may serve as markers of disease progression. 4) Use of genetic analysis, proteomics, RNA transcriptomics and metabolomics for the identification of novel genetic factors and/or biomarkers that correlate with clinically relevant COPD subtypes in individuals with COPD and with one or more of the markers of disease progression.

\section{Study design}

ECLIPSE is an ongoing 3-yr noninterventional longitudinal prospective study being conducted at 46 centres in 12 countries. Following a baseline visit, subjects are to be followed-up at a total of seven visits at: 3 months, 6 months and every 6 months thereafter for 3 yrs. In addition to the study visits, COPD patients are to be telephoned each month between clinic visits in order to assess exacerbation rates. All subjects are to continue to receive their normal prescribed medications throughout the study; no medications are prohibited during the study. The study is being conducted in accordance with the Declaration of Helsinki and good clinical practice guidelines, and has been approved by the relevant ethics and review boards at the participating centres.

\section{Subject participation}

A planned total of 2,180 COPD patients aged 40-75 yrs, with baseline post-bronchodilator FEV1 of $<80 \%$ of the predicted value, baseline post-bronchodilator FEV1/forced vital capacity (FVC) of $\leqslant 0.7$ and a smoking history of $\geqslant 10$ pack-yrs are to be enrolled. In addition, 566 control subjects (post-bronchodilator FEV1 of $>85 \%$ pred and FEV1/FVC of $>0.7$ ) aged $40-75$ yrs are to be recruited, forming two groups: 343 controls with a smoking history of $\geqslant 10$ pack-yrs, and 223 nonsmoking controls with a smoking history of $<1$ pack-yr. The inclusion criteria for COPD patients and control subjects are shown in table 1. Exclusion criteria were related to diagnosis (known respiratory disorders other than COPD and severe $\alpha_{1}$-antitrypsin deficiency), prior medical history (known history of significant inflammatory disease other than COPD, a COPD exacerbation within 4 weeks of enrolment, having undergone lung surgery, recent diagnosis of cancer, having received a blood transfusion in the 4 weeks prior to study start, inability to walk, taking part in a blinded drug study, therapy with oral corticosteroids at inclusion and participation in studies with radiation exposure. In addition, the usual criteria of serious uncontrolled disease likely to interfere with the study or impact on subject safety and substance abuse were applied.

\section{Outcome measurements}

End-points measured in the ECLIPSE study include lung physiology, imaging, biomarkers, health outcomes and genetics. The end-points are listed in table 2; they are not listed in order of importance.

Measures of lung physiology and imaging are central parameters. All subjects are to undergo spirometry and impulse oscillometry at all study visits. Body plethysmography measurements are to be performed annually at selected sites, with recording of static lung volumes, airway resistance and specific conductance. Biomarkers in blood are to be assessed at all sites, whereas sputum, urine and exhaled breath condensate are to be collected at selected sites. At baseline and after 1 and 3 yrs, all subjects are to undergo a low-dose volumetric CT scan (120 kV peak, $40 \mathrm{~mA}$ and 1.00 or 1.25 -mm slice thickness) at full inspiration. The radiation dose is estimated to be $1.67 \mathrm{mSv}$ per CT study or $5 \mathrm{mSv}$ for the entire ECLIPSE protocol. All scans are to be sent for evaluation at the central imaging unit at the University of British Columbia (Vancouver, BC, Canada).

\section{Study organisation}

The present study is guided by a Steering Committee, consisting of five academic physicians and representatives of the study sponsor, GlaxoSmithKline. A Scientific Committee of six academic investigators reports to the steering committee on specific scientific issues and proposals for substudies.

\section{DISCUSSION}

COPD is characterised by abnormal spirometric results, with a focus on FEV1. Since small airways disease is an important component of COPD, and FEV1 is not very sensitive to changes in small airway calibre, other measures may more accurately reflect small airway function. In addition, FEV1 is insensitive to the severity of emphysema in COPD, and patients with similar FEV1 may exhibit very different underlying pathologies. As an assessment of severity, FEV1 correlates poorly with clinical 
TABLE 1 Inclusion criteria for the Evaluation of COPD Longitudinally to Identify Predictive Surrogate End-points (ECLIPSE) study

Male/female subjects aged 40-75 yrs

Baseline post-bronchodilator FEV1 of $<80 \%$ pred and $\mathrm{FEV}_{1} / \mathrm{FVC}$ of $\leqslant 0.7$

Current or ex-smokers with a smoking history of $\geqslant 10$ pack-yrs ${ }^{\#}$

Signed and dated written informed consent obtained prior to participation

Ability to comply with the requirements of the protocol and be available for study visits over 3 yrs
Male/female subjects aged 40-75 yrs, who are free from significant disease as determined by history, physical examination and screening investigations

Baseline post-bronchodilator FEV 1 of $>85 \%$ pred and $\mathrm{FEV}_{1} / \mathrm{FVC}$ of $>0.7$

Current or ex-smokers with a smoking history $\geqslant 10$ pack-yrs ${ }^{\#}$

Signed and dated written informed consent obtained prior to participation

Ability to comply with the requirements of the protocol and be available for study visits over 3 yrs
Male/female subjects aged $40-75$ yrs, who are free from significant disease as determined by history, physical examination and screening investigations

Baseline post-bronchodilator FEV 1 of $>85 \%$ pred and FEV $1 / F V C$ of $>0.7$

Nonsmokers with a smoking history of $<1$ pack-yr

Signed and dated written informed consent obtained prior to participation

Ability to comply with the requirements of the protocol and be available for study visits over 3 yrs

COPD: chronic obstructive pulmonary disease; FEV1: forced expiratory volume in one second; \% pred: \% predicted; FVC: forced vital capacity. ${ }^{\#}$ : equivalent to, for example, 20 cigarettes $\cdot$ day $^{-1}$ for $10 \mathrm{yrs}$ or 10 cigarettes $\cdot$ day $^{-1}$ for $20 \mathrm{yrs} .1$ pack-yr=1 pack (20 cigarettes) $\cdot$ day $^{-1}$ for $1 \mathrm{yr}$.

parameters (e.g. dyspnoea, quality of life and cost of care). In order to follow COPD over time, studies using decline in FEV1 for the assessment of COPD progression typically require $\geqslant 3 \mathrm{yrs}$, and the costs and efforts associated with studies of $\geqslant 3$ yrs duration limit the number of novel drugs that can be considered for disease modification. Finally, although FEV1, as well as other measures of lung function, can be used for prognostic purposes, the association between mortality and FEV1 is considerably stronger when other measures are added to the predictive equation [11]. Clearly, more sensitive measures of assessing COPD severity and disease progression are needed. Such measures should ideally reflect several components, e.g. inflammation, structural changes, disease activity, impact on patients' lives and prognosis.

Airway inflammation is a key component of the pathogenesis of COPD $[5,6]$. The inflammation can be characterised by various differential cell profiles, which may reflect different inflammatory subgroups $[12,13]$, as well as increased levels of various inflammatory mediators [14, 15]. Airway inflammation can, to some extent, be studied noninvasively using induced sputum and exhaled breath condensate, and both measures are being applied to subsets of the ECLIPSE population. Systemic inflammation is increasingly being recognised as an important feature of COPD [16]. To date, C-reactive protein has been reviewed most extensively; however, a number of other markers may be of importance, and profiling of systemic inflammation is important in the ECLIPSE study.

CT scanning is a crucial element of the ECLIPSE study for the determination of different pathogenic phenotypes. Three lowdose volumetric $\mathrm{CT}$ scans and quantitative analyses are to be conducted as a tool for dividing COPD into subgroups based on structural changes and evaluation of disease progression. CT lung density measurements are to be used for the evaluation of both degree and distribution of emphysema and airway wall dimensions [17]. This area is clearly not yet fully developed, but previous data suggest that risk factors for COPD may differ depending upon the identification of emphysema/airway remodelling on CT.

Exacerbations are important events influencing disease severity, health-related quality of life, disease progression and mortality [18], and characterisation of frequency, type and duration of exacerbation could be of significant importance. In the ECLIPSE study, all subjects are contacted monthly by telephone, employing a structured interview scheme in order to capture exacerbations. Other more detailed tools, such as diary cards, may have been more accurate for this characterisation but were considered unfeasible in a population of this size from several different countries.

Various other health outcome measures have been correlated with COPD progression. Breathlessness, which in the ECLIPSE study is measured using a modified UK Medical Research Council dyspnoea scale, is an independent predictor of mortality [9]. The same is the case for St George's Respiratory Questionnaire scores [19] and exercise capacity [20]. In general, it is unlikely that any single outcome measure would accurately predict clinical progression in all COPD patients; the ECLIPSE study aims to examine whether the body mass index, airflow obstruction, dyspnoea and exercise capacity index [11] or other prognostic indices may be of value across different COPD subtypes.

Comorbid conditions in COPD are of importance since they are frequent and affect prognosis as well as costs of COPD [21]. In the ECLIPSE study, it was decided not to exclude subjects with cardiovascular comorbid conditions unless they were of a severity that made it unlikely that the patients could complete a 3-yr study. It was, however, decided to exclude diseases with significant systemic inflammation, such as rheumatoid arthritis and inflammatory bowel disease. The present authors are aware that this could potentially introduce a bias or at least reduce the generalisability of the ECLIPSE findings somewhat, 
TABLE 2 Outcomes measured in the Evaluation of COPD Longitudinally to Identify Predictive Surrogate End-points (ECLIPSE) study

\section{Pulmonary function}

Lung function: post-bronchodilator $F E V_{1}$, rate of decline

Each visit

in FEV1, FVC, FEV1/FVC, FEV6, SVC and reversibility

Pulmonary plethysmography: RV, TLC, FRC, airway resistance and specific conductance

Impulse oscillometry: frequency-dependent resistance and reactance parameters

Exhaled carbon monoxide

Whole body impedance/fat-free mass

Body composition

Fat-free mass

Chest computed tomography

\section{Exercise capacity ${ }^{+}$ \\ Resting oxygen saturation \\ Biomarkers}

Blood samples (for protein or mRNA): CRP, TNF- $\alpha$,

IL-6, Clara cell protein, IL-8 and surfactant protein D

Induced sputum : inflammatory cell content,

soluble markers (e.g. myeloperoxidase) and cellular expression of mRNA

Exhaled breath condensate

Blood and urine metabolomics

\section{Health outcomes}

Exacerbation assessment

ATS respiratory questionnaire

Depression questionnaire

Fatigue questionnaire

Health status and MRC dyspnoea assessment

\section{Blood samples for genetic markers}

DNA
Measurement of peripheral airways function during tidal breathing

Confirmation of smoking status

Estimated using single-frequency $(50 \mathrm{kHz})$ bioelectrical impedance analysis

Calculated from height squared/impedance, age, sex and body weight

Measurement of airway dimensions and quantification of emphysema; to be used for patients stratification during data analysis

Supervised standardised 6-min walking test

Measured after 10-min rest

Evaluation of association with disease subtypes and their relationship with disease progression

Measured using open-platform technologies (transcriptomics and proteomics) or specific validated assays (ELISA, multiplex and quantitative reverse transcriptase PCR)

Total and differential cell count, supernatant proteomics and cell extract transcriptomics

Collected by the cooling and freezing of spontaneously exhaled air

Taken after 3-h fast following completion of food intake diary

Details of doctor/hospital visits and use of oral corticosteroids/ antibiotics recorded

Annually

Each visit

Each visi

Annually

Annually

Years 1 and 3

Annually

Each visit

Each visit

Annually

Year 3

Each visit

Each visit plus monthly phone calls

Standardised ATS epidemiology questionnaire

CES-D scale

FACIT fatigue scale

SGRQ-C, modified MRC dyspnoea scale, BODE index and Prognostic Index

Identification and/or confirmation of genes believed to be associated with COPD-related phenotypes and COPD subtypes
Baseline alone

Year 3

Year 3

Annually

Ongoing

COPD: chronic obstructive pulmonary disease; FEV1: forced expiratory volume in one second; FVC: forced vital capacity; FEV6: forced expiratory volume in six seconds: SVC: slow vital capacity; RV: residual volume; TLC: total lung capacity; FRC: functional residual capacity; CRP: C-reactive protein; TNF: tumour necrosis factor; IL: interleukin ATS: American Thoracic Society; MRC: UK Medical Research Council; CES-D: Centre for Epidemiological Studies of Depression; FACIT: Functional Assessment of Chronic IIIness Therapy; SGRQ-C: COPD-specific version of the St George's Respiratory Questionnaire; BODE: body mass index, airflow obstruction, dyspnoea and exercise capacity. ${ }^{*}$ : in addition to baseline; ": at selected sites, involving 500-510 individuals; ${ }^{+}$: COPD patients alone; ${ }^{\text {s }}$ : baseline alone in nonsmoking controls

but would prefer to run that risk rather than contaminate the population with non-COPD-related systemic inflammation.

The major dilemma of a study such as ECLIPSE, which is aimed at finding surrogate markers superior to FEV1, is the fact that the gold standard for both the diagnosis and assessment of the rate of progression is currently FEV1. The challenge of the ECLIPSE study is, therefore, to utilise the various information gathered to describe models of COPD rooted in the classic definition of COPD, but with much more detail and the ability to capture the dynamics of the different processes that result in the clinical subgroups that eventually make up the syndrome nowadays called COPD. It is recognised that putative surrogate end-points identified from the ECLIPSE study would need to be further tested in subsequent studies.

In conclusion, the Evaluation of COPD Longitudinally to Identify Predictive Surrogate End-points study is the first study in a large number of subjects with the primary objective of describing the subtypes of chronic obstructive pulmonary 
disease, defining predictive or surrogate markers of disease progression, and possibly identifying novel targets for therapeutic intervention.

\section{ACKNOWLEDGEMENTS}

The members of the Evaluation of COPD Longitudinally to Identify Predictive Surrogate End-points (ECLIPSE) Steering Committee are: H. Coxson (University of British Columbia, Vancouver, BC, Canada); L. Edwards (GlaxoSmithKline, Research Triangle Park, NC, USA); K. Knobil (Co-Chair; GlaxoSmithKline, Greenford, UK); D. Lomas (University of Cambridge, Cambridge, UK); W. MacNee (University of Edinburgh, Edinburgh, UK); E. Silverman (Brigham and Women's Hospital, Boston, MA, USA); R. Tal-Singer (GlaxoSmithKline, King of Prussia, PA, USA); J. Vestbo (CoChair; Hvidovre Hospital, Hvidovre, Denmark); and J. Yates (GlaxoSmithKline, Research Triangle Park).

The members of the ECLIPSE Scientific Committee are: A. Agusti (Son Dureta Hospital and Cimera, Palma, Spain); P. Calverley (University Hospital Aintree, Liverpool, UK); B. Celli (Caritas St. Elizabeth's Medical Center, Boston, MA, USA); C. Crim (GlaxoSmithKline, Research Triangle Park); G. Hagan (GlaxoSmithKline, Greenford); W. MacNee (Chair); S. Rennard (University of Nebraska, Omaha, NE, USA); R. TalSinger, E. Wouters (University of Maastricht, Maastricht, the Netherlands); and J. Yates.

\section{REFERENCES}

1 World Health Organization. The World Health Report 2004: Changing History. www.who.int/whr/2004/en/ Date last accessed: January 21, 2008.

2 Lopez AD, Shibuya K, Rao C, et al. Chronic obstructive pulmonary disease: current burden and future projections. Eur Respir J 2006; 27: 397-412.

3 Agusti AG. COPD, a multicomponent disease: implications for management. Respir Med 2005; 99: 670-682.

4 Laperre TS, Snoeck-Stroband JB, Gosman MM, et al. Dissociation of lung function and airway inflammation in chronic obstructive pulmonary disease. Am J Respir Crit Care Med 2004; 170: 499-504.

5 Rabe KF, Hurd S, Anzueto A, et al. Global strategy for the diagnosis, management, and prevention of chronic obstructive pulmonary disease. GOLD executive summary. Am J Respir Crit Care Med 2007; 176: 532-555.

6 Celli BR, MacNee W, ATS/ERS Task Force. Standards for the diagnosis and treatment of patients with COPD: a summary of the ATS/ERS position paper. Eur Respir J 2004; 23: 932-946.

7 Franciosi LG, Page CP, Celli BR, et al. Markers of disease severity in chronic obstructive pulmonary disease. Pulm Pharmacol Ther 2006; 19: 189-199.
8 Gelb AF, Hogg JC, Müller NL, et al. Contribution of emphysema and small airways in COPD. Chest 1996; 109: 353-359.

9 Nishimura K, Izumi T, Tsukino M, Oga T. Dyspnea is a better predictor of 5-year survival than airway obstruction in patients with COPD. Chest 2002; 121: 1434-1440.

10 National Institutes of Health. ClinicalTrials.gov. Evaluation of COPD (chronic obstructive pulmonary disease) to Longitudinally Identify Predictive Surrogate Endpoints (ECLIPSE). http:/ / clinicaltrials.gov/ct2/show / NCT00292552?term $=00292552 \&$ rank=1 Date last updated: November 21, 2007. Date last accessed: January 21, 2008.

11 Celli BR, Cote CG, Marin JM, et al. The body-mass index, airflow obstruction, dyspnea, and exercise capacity index in chronic obstructive pulmonary disease. $N$ Engl J Med 2004; 350: 1005-1012.

12 Perng DW, Huang HY, Chen HM, Lee YC, Perng RP. Characteristics of airway inflammation and bronchodilator reversibility in COPD: a potential guide to treatment. Chest 2004; 126: 375-381.

13 Brightling CE, McKenna S, Hargadon B, et al. Sputum eosinophilia and the short term response to inhaled mometasone in chronic obstructive pulmonary disease. Thorax 2005; 60: 193-198.

14 Keatings VM, Collins PD, Scott DM, Barnes PJ. Differences in interleukin- 8 and tumor necrosis factor- $\alpha$ in induced sputum from patients with chronic obstructive pulmonary disease or asthma. Am J Respir Crit Care Med 1996; 153: 530-534.

15 Woolhouse IS, Bayley DL, Stockley RA. Sputum chemotactic activity in chronic obstructive pulmonary disease: effect of $\alpha_{1}$-antitrypsin deficiency and the role of leukotriene $\mathrm{B}_{4}$ and interleukin-8. Thorax 2002; 57: 709-714.

16 Gan WQ, Man SF, Senthilselvan A, Sin DD. Association between chronic obstructive pulmonary disease and systemic inflammation: a systematic review and a metaanalysis. Thorax 2004; 59: 574-580.

17 Hansell DM. Small airways diseases: detection and insights with computed tomography. Eur Respir J 2001; 17: 1294-1313.

18 Donaldson GC, Wedzicha JA. COPD exacerbations. 1: Epidemiology. Thorax 2006; 61: 164-168.

19 Domingo-Salvany A, Lamarca R, Ferrer M, et al. Healthrelated quality of life and mortality in male patients with chronic obstructive pulmonary disease. Am J Respir Crit Care Med 2002; 166: 680-685.

20 Oga T, Nishimura K, Tsukino M, Sato S, Hajiro T. Analysis of the factors related to mortality in chronic obstructive pulmonary disease: role of exercise capacity and health status. Am J Respir Crit Care Med 2003; 167: 544-549.

21 Viegi G, Pistelli F, Sherrill DL, Maio S, Baldacci S, Carrozzi L. Definition, epidemiology and natural history of COPD. Eur Respir J 2007; 30: 993-1013. 